\title{
Solar-Sail Navigation: Estimation of Force, Moments, and Optical Parameters
}

\author{
L. Rios-Reyes** and D. J. Scheeres \\ University of Michigan, Ann Arbor, Michigan 48109-2140
}

DOI: $10.2514 / 1.24340$

\begin{abstract}
Determining a solar sail's mathematical model on the ground is an inherently difficult task. Uncertainties in the sail shape are always present because the sail's precise curvature and surface structure may change after deployment in space. These changes may invalidate previous measurements and computations. For accurate navigation, it is essential that the postlaunch sail mathematical model is estimated. In this paper, we provide a methodology for estimating the force and moment generated by a sail of arbitrary, but constant, shape based on in-flight data. This is accomplished by finding the force and moment tensor coefficients defined by the generalized sail model, which analytically models the characteristics of solar sails with arbitrary shapes. Several data sampling examples are studied to determine the attitudes that allow for better estimation of the sail's force and moment coefficients.
\end{abstract}

\section{Introduction}

A $\mathrm{S}$ WITH all space missions, success depends on having a precise spacecraft mathematical model. This is also true for solar sails and is a capability needed for them because several of the world's space agencies are considering solar sails for space missions. Initial mission planning can be performed based on data from ground measurements. However, due to the complexity of sail shapes a refinement of the sail propulsion model will be necessary after launch for navigating the sail to its target. Sail wrinkles after in-space deployment, deformation from nominal shape, and surface degradation due to the space environment [1] are some of the effects that make prelaunch sail propulsion determination inaccurate.

The recently developed generalized sail model [2], GSM, allows for the analytic computation of forces and moments acting on a solar sail of arbitrary fixed shape. These forces and moments are computed analytically using a set of tensor coefficients, which for constant optical parameters are defined solely by the sail shape. The GSM assumes that the sail shape remains fixed with attitude; however small changes in the sail shape due to attitude should not affect the estimation results as the estimation is not on the sail shape but on the GSM tensor coefficients. If the sail remains in a fixed attitude for a given period of time, the sail shape is expected to change due to thermal effects [3], and although accurate sail shape models can be obtained in ground tests [4], sails with varying geometry should be further studied when more data are available on how these changes take place. Despite these limitations, we believe the changes to the estimated coefficients will be small and future work on this subject will start from our current analysis. The coefficients for computing the force number 19 arranged in three tensors. The moment computation requires at most 27 coefficients arranged in two tensors.

The generalized equations for the sail force and moment are linear in the GSM coefficients. This fact and its analytic nature allows us to develop linear estimation methods for these coefficients using measured accelerations and moments as input data. The force and

Presented as Paper 6225 at the 16th AAS/AIAA Space Fligh Mechanics Meetings, Tampa, FL, 22-26 January 2006; received 29 March 2006; revision received 4 October 2006; accepted for publication 9 October 2006. Copyright ( 2006 by Leonel Rios-Reyes and Daniel J. Scheeres. Published by the American Institute of Aeronautics and Astronautics, Inc., with permission. Copies of this paper may be made for personal or internal use, on condition that the copier pay the $\$ 10.00$ per-copy fee to the Copyright Clearance Center, Inc., 222 Rosewood Drive, Danvers, MA 01923; include the code 0731-5090/07 \$10.00 in correspondence with the CCC.

*Post-Doctoral Researcher, Department of Aerospace Engineering, 1320 Beal Avenue; leonelr@umich.edu. Student Member AIAA, Student Member AAS.

${ }^{\dagger}$ Associate Professor, Department of Aerospace Engineering, 1320 Beal Avenue; scheeres@umich.edu. Member AAS, Associate Fellow AIAA. moment tensor coefficients are estimated using a least-squares algorithm from simulated navigation data and predictions from finite element models of solar sails. The estimation results are highly dependent on the attitudes at which the sail navigation data are taken. It is not only important to take measurements at several different sunrelative attitudes, but also to find those attitudes that capture the geometry of the sail. The accuracy of the estimates will vary for different data sampling strategies. Here, we present several data sampling examples and comment on their impact to the accuracy of the estimation results.

The sail surface degrades due to the solar radiation and space environment [1] making the optical parameters change with time. Thus, estimating the GSM coefficients with fixed optical parameters may lead to inaccurate results. It may be possible to estimate both the optical parameters and the GSM coefficients, but this would require a nonlinear estimation method or an iterative procedure. Instead, by including the optical parameters into the definition of the tensor coefficients, the estimation can be done on the combination of the optical parameters and tensor coefficients. This would allow for tracking changes on the sail properties over time through the tensor coefficients.

\section{Solar-Sail Modeling}

The flat sail model is derived in [5]. This model captures nonideal optical parameters and decomposes the force into normal and transverse components:

$$
\begin{array}{r}
\mathbf{F}_{n}=P(r) A\left[(1+\rho s) \cos ^{2} \alpha+B_{f}(1-s) \rho \cos \alpha\right. \\
\left.+(1-\rho) \frac{\epsilon_{f} B_{f}-\epsilon_{b} B_{b}}{\epsilon_{f}+\epsilon_{b}} \cos \alpha\right] \hat{\mathbf{n}} \\
\mathbf{F}_{t}=P(r) A(1-\rho s) \cos \alpha \sin \alpha \hat{\mathbf{t}}
\end{array}
$$

where $P$ is the solar radiation pressure at a distance $r$ from the sun as derived in [5], $A$ is the sail area, $\rho$ is the reflectivity, $s$ is the fraction of specular reflection, $B$ and $\epsilon$ are the Lambertian coefficient and the surface emissivity with the subscripts $f$ and $b$ denoting the front and back sides of the sail, $\alpha$ is the sun-sail line angle, $\hat{\mathbf{n}}$ is the sail normal vector, and $\hat{\mathbf{t}}$ is the transverse vector.

The generalized sail model is a generalization of the flat sail model for arbitrary shapes. Deviations from flatness in the sail membrane are captured in tensors obtained by performing a surface integration of all the differential forces generated by the differential areas. Here, it is assumed that the sail shape remains fixed, irrespective of attitude, and that no self-shadowing occurs so that all the sail surface remains 
illuminated all the time. With these assumptions, when this surface integration is performed, the total force in the sail body fixed frame is given by [2]

$$
\mathbf{F}=P(r) A\left[\mathbf{J}^{2} \cdot \hat{\mathbf{r}}-2 \hat{\mathbf{r}} \cdot \mathbf{J}^{3} \cdot \hat{\mathbf{r}}-\left(\mathbf{J}^{1} \cdot \hat{\mathbf{r}}\right) \hat{\mathbf{r}}\right]
$$

where $\hat{\mathbf{r}}$ is the sun's unit position vector from the sun into the sail. The vectors $\hat{\mathbf{r}}, \hat{\mathbf{n}}$, and $\hat{\mathbf{t}}$ lie in the same plane. $\hat{\mathbf{n}}$ and $\hat{\mathbf{t}}$ are orthogonal to each other. $\mathbf{J}^{m}$ is a rank- $m$ force tensor defined as integrals over the surface area [2]:

$$
\begin{gathered}
\mathbf{J}^{1}=\frac{1}{A} \int_{A} a_{3} \hat{\mathbf{n}} \mathrm{d} A \\
\mathbf{J}^{2}=\frac{1}{A} \int_{A} a_{2} \hat{\mathbf{n}} \hat{\mathbf{n}} \mathrm{d} A \\
\mathbf{J}^{3}=\frac{1}{A} \int_{A} \rho s \hat{\mathbf{n}} \hat{\mathbf{n}} \hat{\mathbf{n}} \mathrm{d} A
\end{gathered}
$$

where

$$
a_{2}=B_{f}(1-s) \rho+(1-\rho) \frac{\epsilon_{f} B_{f}-\epsilon_{b} B_{b}}{\epsilon_{f}+\epsilon_{b}}
$$

$a_{3}=1-\rho s$, and $\hat{\mathbf{n}}$ is a function of location on the sail. This general result captures arbitrary optical parameters across the sail surface by including them inside the integrals. Because the force tensors are defined as integrals of the outer product of the same vector $(\hat{\mathbf{n}})$, they are symmetrical in all their indices, that is, $\mathbf{J}_{i \cdots j \cdots k}^{m}=\mathbf{J}_{k \cdots i \cdots j}^{m}$, hence only 19 parameters are needed to specify the force on a sail of arbitrary shape.

The differential moment acting on the sail due to a differential sail element is

$$
\mathrm{d} \mathbf{M}=\varrho \times \mathrm{d} \mathbf{F}=\tilde{\varrho} \cdot \mathrm{d} \mathbf{F}
$$

where $\varrho$ is the position vector of the differential element $\mathrm{d} A$ with respect to a given reference frame on the sail. The operator ( $\left.{ }^{\sim}\right)$ allows us to write the cross product as the product of a matrix and a vector, by arranging the elements of the vector $\varrho$ into a skew-symmetric matrix:

$$
\tilde{\varrho}=\left[\begin{array}{ccc}
0 & -\varrho_{3} & \varrho_{2} \\
\varrho_{3} & 0 & -\varrho_{1} \\
-\varrho_{2} & -\varrho_{1} & 0
\end{array}\right]
$$

Thus, the differential moment acting on the sail is just

$$
\begin{aligned}
& \mathrm{d} \mathbf{M}=P(r)\left[a_{2} \tilde{\varrho} \cdot \hat{\mathbf{n}} \hat{\mathbf{n}} \mathrm{d} A \cdot \hat{\mathbf{r}}+\hat{\mathbf{r}} \cdot\left(-2 \rho s \tilde{\varrho} \cdot \hat{\mathbf{n}} \hat{\mathbf{n}} \hat{\mathbf{n}} \mathrm{d} A-a_{3} \tilde{\varrho}\right.\right. \\
&\cdot \hat{\mathbf{n}} \overline{\overline{\mathbf{U}}} \mathrm{d} A) \cdot \hat{\mathbf{r}}]
\end{aligned}
$$

where $\overline{\overline{\mathbf{U}}}$ is the identity dyad.

The moment integrals can be defined as

$$
\begin{gathered}
\mathbf{K}^{2}=\frac{1}{A l_{r}} \int_{A} a_{2} \tilde{\varrho} \cdot \hat{\mathbf{n}} \hat{\mathbf{n}} \mathrm{d} A \\
\mathbf{K}^{3}=\frac{1}{A l_{r}} \int_{A}[\rho s(-2 \tilde{\varrho} \cdot \hat{\mathbf{n}} \hat{\mathbf{n}} \hat{\mathbf{n}}+\tilde{\varrho} \cdot \hat{\mathbf{n}} \overline{\overline{\mathbf{U}}})-\tilde{\varrho} \cdot \hat{\mathbf{n}} \overline{\overline{\mathbf{U}}}] \mathrm{d} A
\end{gathered}
$$

where $l_{r}$ is an arbitrary reference length. The total moment is just

$$
\mathbf{M}=P A l_{r}\left[\mathbf{K}^{2} \cdot \hat{\mathbf{r}}+\hat{\mathbf{r}} \cdot \mathbf{K}^{3} \cdot \hat{\mathbf{r}}\right]
$$

$\mathbf{K}^{3}$ is a rank-3 tensor symmetric in its last two indices, that is, $\mathbf{K}_{i j k}^{3}=\mathbf{K}_{i k j}^{3}$, since two $\hat{\mathbf{n}}$ vectors appear inside the integral, requiring 18 coefficients. Thus, only 27 parameters are needed to capture the moment being generated by an arbitrary sail. Note that this is a more compact definition than defined previously in [2].

\section{Linear Estimation of GSM Tensor Coefficients}

To correct inaccuracies in the prelaunch prediction of the sail model performance, we wish to estimate the GSM tensor coefficients from navigation or initial data provided from detailed measurements or simulations. In the following the sail force and moments are reported in a nondimensional form by dividing the force values by the solar radiation pressure and total sail area and the moments by an additional reference length $l_{r}$ [ $\underline{6}$. Thus, the force and moment vectors can be written as

$$
\begin{gathered}
\mathbf{F}=P A \mathbf{C}_{F} \\
\mathbf{M}=P A l_{r} \mathbf{C}_{M}
\end{gathered}
$$

where $\mathbf{C}$ is a vector containing the normalized values from actual navigation data or tested data with the subscripts denoting force or moment.

In the following we assume that the navigation data are reported as normalized force and moment vectors. The normalized version of the GSM equations are

$$
\begin{gathered}
\mathbf{F}_{c}=\mathbf{J}^{2} \cdot \hat{\mathbf{r}}-2 \hat{\mathbf{r}} \cdot \mathbf{J}^{3} \cdot \hat{\mathbf{r}}-\left(\mathbf{J}^{1} \cdot \hat{\mathbf{r}}\right) \hat{\mathbf{r}} \\
\mathbf{M}_{c}=\mathbf{K}^{2} \cdot \hat{\mathbf{r}}+\hat{\mathbf{r}} \cdot \mathbf{K}^{3} \cdot \hat{\mathbf{r}}
\end{gathered}
$$

Force and moment can be obtained from measurements taken through on-board instruments such as inertial measurement units, gyroscopes, and with ground tracking. Note that the measured onboard rotational accelerations must be related, via the moment of inertia, to the total moment acting on the sailcraft. We do not consider the estimation of the inertia tensor or sailcraft mass in our current work.

\section{A. Force and Moment in Linear Form}

The normalized force and moment are linear in all the coefficients of the $\mathbf{J}^{m}$ and $\mathbf{K}^{m}$ tensors, thus a least-squares estimation can be easily employed for finding the best values of the tensor coefficients. But before this step is done, it is necessary to manipulate the equations and write them in a more useful way as the product of a matrix and a vector. The matrix contains information on the sail attitude whereas the vector contains the tensor coefficients that need to be estimated. Thus, the normalized force can be written as

$$
\mathbf{F}_{c}=\mathbf{A}_{F}(\hat{\mathbf{r}}) \mathbf{J}
$$

where the matrix $\mathbf{A}_{F}(\hat{\mathbf{r}})$ is a function of the sail attitude alone, and $\mathbf{J}$ is a $19 \times 1$ vector containing the independent force tensor coefficients. The force matrix is just

$$
\mathbf{A}_{F}=\left[\begin{array}{lll}
-\mathbf{A}_{F 1} & \mathbf{A}_{F 2} & -2 \mathbf{A}_{F 3}
\end{array}\right]
$$

where

$$
\mathbf{A}_{F 1}=\left[\begin{array}{ccc}
\hat{r}_{1}^{2} & \hat{r}_{1} \hat{r}_{2} & \hat{r}_{1} \hat{r}_{3} \\
\hat{r}_{1} \hat{r}_{2} & \hat{r}_{2}^{2} & \hat{r}_{2} \hat{r}_{3} \\
\hat{r}_{1} \hat{r}_{3} & \hat{r}_{2} \hat{r}_{3} & \hat{r}_{3}^{2}
\end{array}\right]
$$

$$
\mathbf{A}_{F 2}=\left[\begin{array}{cccccc}
\hat{r}_{1} & \hat{r}_{2} & \hat{r}_{3} & 0 & 0 & 0 \\
0 & \hat{r}_{1} & 0 & \hat{r}_{2} & \hat{r}_{3} & 0 \\
0 & 0 & \hat{r}_{1} & 0 & \hat{r}_{2} & \hat{r}_{3}
\end{array}\right]
$$




$$
\mathbf{A}_{F 3}=\left[\begin{array}{cccccccccc}
\hat{r}_{1}^{2} & 2 \hat{r}_{1} \hat{r}_{2} & \hat{r}_{2}^{2} & 2 \hat{r}_{2} \hat{r}_{3} & 2 \hat{r}_{1} \hat{r}_{3} & \hat{r}_{3}^{2} & 0 & 0 & 0 & 0 \\
0 & \hat{r}_{1}^{2} & 2 \hat{r}_{1} \hat{r}_{2} & 2 \hat{r}_{1} \hat{r}_{3} & 0 & 0 & \hat{r}_{2}^{2} & 2 \hat{r}_{2} \hat{r}_{3} & \hat{r}_{3}^{2} & 0 \\
0 & 0 & 0 & 2 \hat{r}_{1} \hat{r}_{2} & \hat{r}_{1}^{2} & 2 \hat{r}_{1} \hat{r}_{3} & 0 & \hat{r}_{2}^{2} & 2 \hat{r}_{2} \hat{r}_{3} & \hat{r}_{3}^{2}
\end{array}\right]
$$

and the force coefficient vector is

$$
\mathbf{J}=\left[\begin{array}{llllllllllllllllllll}
J_{1}^{1} & J_{2}^{1} & J_{3}^{1} & J_{11}^{2} & J_{12}^{2} & J_{13}^{2} & J_{22}^{2} & J_{23}^{2} & J_{33}^{2} & J_{111}^{3} & J_{121}^{3} & J_{221}^{3} & J_{231}^{3} & J_{131}^{3} & J_{331}^{3} & J_{222}^{3} & J_{232}^{3} & J_{332}^{3} & J_{333}^{3}
\end{array}\right]^{T}
$$

Similarly, the moment equation also can be written as the product of a matrix, which contains information on the sail attitude at which the measurements are taken, and a vector, which includes the information of the moment tensor coefficients:

$$
\mathbf{M}_{c}=\mathbf{A}_{M} \mathbf{K}
$$

where

$$
\begin{aligned}
& \mathbf{A}_{M}=\left[\begin{array}{ll}
\mathbf{A}_{M 2} & \mathbf{A}_{M 3}
\end{array}\right] \\
& \mathbf{A}_{M 2}=\left[\begin{array}{ccccccccc}
\hat{r}_{1} & \hat{r}_{2} & \hat{r}_{3} & 0 & 0 & 0 & 0 & 0 & 0 \\
0 & 0 & 0 & \hat{r}_{1} & \hat{r}_{2} & \hat{r}_{3} & 0 & 0 & 0 \\
0 & 0 & 0 & 0 & 0 & 0 & \hat{r}_{1} & \hat{r}_{2} & \hat{r}_{3}
\end{array}\right] \\
& \mathbf{A}_{M 3}=\left[\begin{array}{cccccccccccccccccc}
\hat{r}_{1}^{2} & \hat{r}_{1} \hat{r}_{2} & \hat{r}_{1} \hat{r}_{3} & \hat{r}_{1} \hat{r}_{2} & \hat{r}_{2}^{2} & \hat{r}_{2} \hat{r}_{3} & \hat{r}_{1} \hat{r}_{3} & \hat{r}_{2} \hat{r}_{3} & \hat{r}_{3}^{2} & 0 & 0 & 0 & 0 & 0 & 0 & 0 & 0 & 0 \\
0 & \hat{r}_{1}^{2} & 0 & 0 & \hat{r}_{1} \hat{r}_{2} & 0 & 0 & \hat{r}_{1} \hat{r}_{3} & 0 & \hat{r}_{1} \hat{r}_{2} & \hat{r}_{1} \hat{r}_{3} & \hat{r}_{2}^{2} & \hat{r}_{2} \hat{r}_{3} & \hat{r}_{2} \hat{r}_{3} & \hat{r}_{3}^{2} & 0 & 0 & 0 \\
0 & 0 & \hat{r}_{1}^{2} & 0 & 0 & \hat{r}_{1} \hat{r}_{2} & 0 & 0 & \hat{r}_{1} \hat{r}_{3} & 0 & \hat{r}_{1} \hat{r}_{2} & 0 & \hat{r}_{2}^{2} & 0 & \hat{r}_{2} \hat{r}_{3} & \hat{r}_{1} \hat{r}_{3} & \hat{r}_{2} \hat{r}_{3} & \hat{r}_{3}^{2}
\end{array}\right]
\end{aligned}
$$

and

$\mathbf{K}$

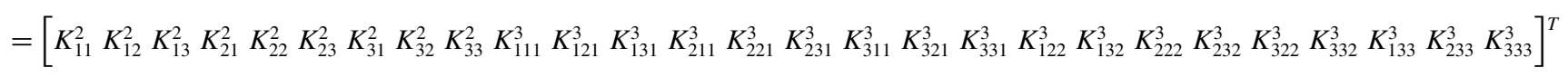

Note for a general sail the force requires 19 parameters and the moment requires 27 . For sails with symmetries the number of force and moment parameters are reduced.

\section{B. Least-Squares Estimation}

For estimating the GSM tensor coefficients, a least-squares cost function is employed with the assumption of $N$ measurements taken at different sun-relative attitudes. A covariance matrix $P_{c c}$, associated with the measurements $\mathbf{y}$, is given by

$$
P_{c c}=\mathbb{E}\left[\left(\mathbf{y}_{j}-\overline{\mathbf{y}}_{j}\right)\left(\mathbf{y}_{j}-\overline{\mathbf{y}}_{j}\right)^{T}\right]
$$

where $\mathbb{E}[]$ is the expected value operator. Ideally

$$
P_{c c}=\left[\begin{array}{ccc}
\sigma_{\omega}^{2} & 0 & 0 \\
0 & \sigma_{\omega}^{2} & 0 \\
0 & 0 & \sigma_{\omega}^{2}
\end{array}\right]
$$

which is the covariance matrix for uncorrelated measurements with variance $\sigma_{\omega}^{2}$. Note that the $\mathbf{y}_{j}$ vectors are either normalized force or moment measurements, depending on the application.

The least-squares cost function is defined as the square of the error between the measurements and the values due to estimated coefficients. Hence it is given by

$$
V=\frac{1}{2} \sum_{j=1}^{N}\left(\mathbf{y}_{j}-\mathbf{A}_{j} \mathbf{x}\right)^{T} P_{c c}^{-1}\left(\mathbf{y}_{j}-\mathbf{A}_{j} \mathbf{x}\right)
$$

where $\mathbf{y}_{j}$ are the normalized vector measurements obtained at an attitude corresponding to $\hat{\mathbf{r}}_{j}, \mathbf{A}_{j}$ is the corresponding matrix to $\mathbf{A}_{F}\left(\hat{\mathbf{r}}_{j}\right)$ or $\mathbf{A}_{M}\left(\hat{\mathbf{r}}_{j}\right)$, and $\mathbf{x}$ is $\mathbf{J}$ or $\mathbf{K}$. The solution is found by minimizing the error with respect to the GSM tensor coefficients as outlined in [7].

Define the information matrix as

$$
\Lambda_{x x}=\sum_{j=1}^{N} \mathbf{A}_{j}^{T} P_{c c}^{-1} \mathbf{A}_{j}
$$

If the information matrix is nonsingular, then a unique estimate can be found. For the force tensor coefficients $\mathbf{A}_{j} \in \mathbb{R}^{3 \times 19}$, thus $\mathbf{A}_{j}^{T} \mathbf{A}_{j}$ has at best rank 3 for an individual measurement. Hence, at least seven measurements are necessary for a unique solution to be feasible. For the moment tensor coefficients $\mathbf{A}_{j} \in \mathbb{R}^{3 \times 27}$, meaning that at least nine measurements are needed for a unique solution. Note that $\hat{\mathbf{r}}_{j}$ must span a rich enough set of values for $\Lambda_{x x}$ to be nonsingular. In particular, for the moment equations if $\hat{r}_{i}=0, \hat{r}_{i}=\hat{r}_{3}$ for $i=1,2$, or $\hat{r}_{1}=\hat{r}_{2}$ over all measurements, we do 
not get a full rank. A trivial example is if the attitude never changes or only switches between a limited set of attitudes. Thus, to ensure a robust solution requires that a full set of attitudes be sampled.

The covariance matrix of the estimates is $P_{x x}=\Lambda_{x x}^{-1}$. Then, the solution of the estimation problem is given by [7]

$$
\mathbf{x}=P_{x x} \sum_{j=1}^{N} \mathbf{A}_{j}^{T} P_{c c}^{-1} \mathbf{y}_{j}
$$

Defining the data vector $\mathbf{Z}$ as

$$
\mathbf{Z}=\sum_{j=1}^{N} \mathbf{A}_{j}^{T} P_{c c}^{-1} \mathbf{y}_{j}
$$

the solution of $\mathbf{x}$ can be expressed as

$$
\mathbf{x}=P_{x x} \mathbf{Z}
$$

Note that the predicted normalized force and moment at an attitude $\hat{\mathbf{r}}$ can then be written in the form

$$
\mathbf{y}=\mathbf{A}(\hat{\mathbf{r}}) P_{x x} \mathbf{Z}
$$

where the matrix $\mathbf{A}(\hat{\mathbf{r}})$ is a function of the attitude and $\mathbf{y}$ is the predicted force or moment.

\section{Parameter Estimation Uncertainty}

Uncertainties in the tensor coefficients translate into force or moment uncertainties. The mapping from tensor coefficients uncertainties into normalized vector uncertainties is given by

$$
\delta \mathbf{y}=\mathbf{A}(\hat{\mathbf{r}}) \delta \mathbf{x}
$$

The covariance of the estimates is obtained by

$$
P_{y y}=\mathbb{E}\left[\delta \mathbf{y} \delta \mathbf{y}^{T}\right]=\mathbf{A}(\hat{\mathbf{r}}) \mathbb{E}\left[\delta \mathbf{x} \delta \mathbf{x}^{T}\right] \mathbf{A}^{T}(\hat{\mathbf{r}})
$$

which is just

$$
P_{y y}=\mathbf{A}(\hat{\mathbf{r}}) P_{x x} \mathbf{A}^{T}(\hat{\mathbf{r}})
$$

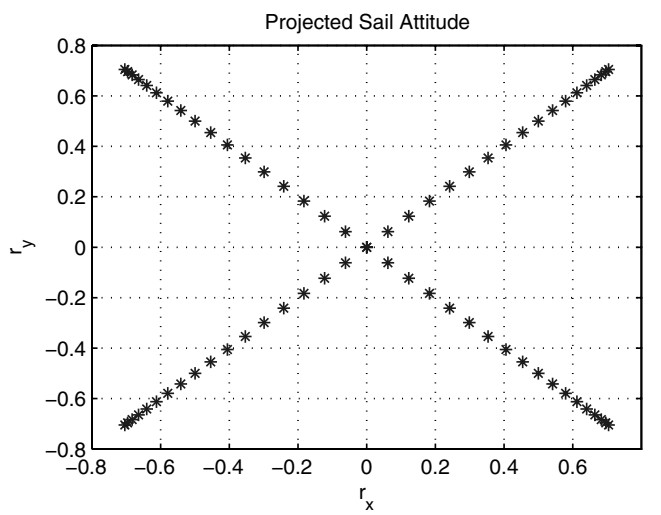

where $P_{x x}$ is the covariance of the estimated GSM tensor coefficients, and equals $\Lambda_{x x}^{-1} . P_{x x}$ allows us to place bounds on the errors of the force and moment estimation. Thus, the force and moment covariance at a given attitude are

$$
\begin{gathered}
P_{F F}=\mathbf{A}_{F}(\hat{\mathbf{r}}) P_{J J} \mathbf{A}_{F}^{T}(\hat{\mathbf{r}}) \\
P_{M M}=\mathbf{A}_{M}(\hat{\mathbf{r}}) P_{K K} \mathbf{A}_{M}^{T}(\hat{\mathbf{r}})
\end{gathered}
$$

respectively.

The results obtained from the least-squares estimation are identical to the results obtained from a best-linear estimation or maximumlikelihood method for the case of normally distributed observations [7].

\section{Numerical Linear Estimation}

Because of the lack of actual navigation data, we define a sail of square planform with fixed general surface and compute forces and moments. The surface of the sail is a sinusoid shape sail with no symmetries. Its exact geometry and computed tensor coefficients are given in the Appendix.

\section{A. Force Estimation}

The results from the estimation are dependent on how the sampling of the measurements is performed as is shown in the following test cases and can be seen from Eqs. (31) and (34) as $\mathbf{A}$ is dependent on the attitude when data are collected. In Figs. 1-4 the covariance of each force tensor coefficient is shown as well as the correlation between them. Four cases with 70 measurements each, but with different measurement sampling attitudes, were used for estimating the tensor coefficients and to compare their respective covariance and correlations as shown in Figs. 1-4. Case I has measurements running along the two diagonals 45 deg from the $x$ and $y$ body-fixed axes. Case II has measurements taken along the $x$ and $y$ body-fixed axes. Case III forms a 4-loop curve in the $x-y$ plane, while Case IV forms a spiral. In practice, the sail would be reoriented to these different attitudes during a characterization phase and the accelerations and moments measured using onboard IMUs (inertial

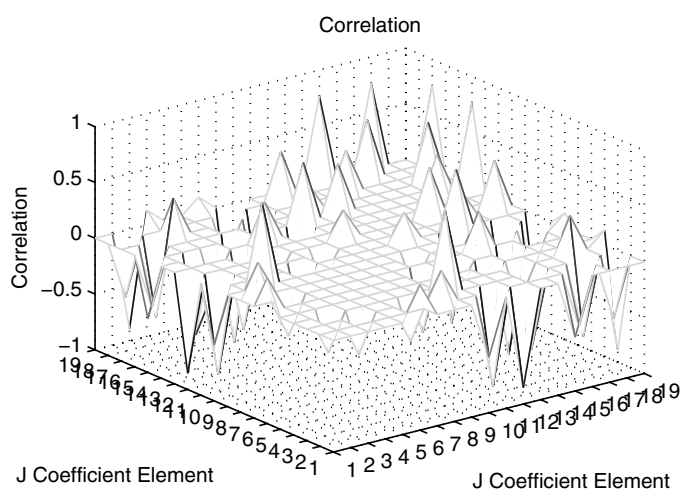

Fig. 1 Attitude, covariance, and correlation, case I. 

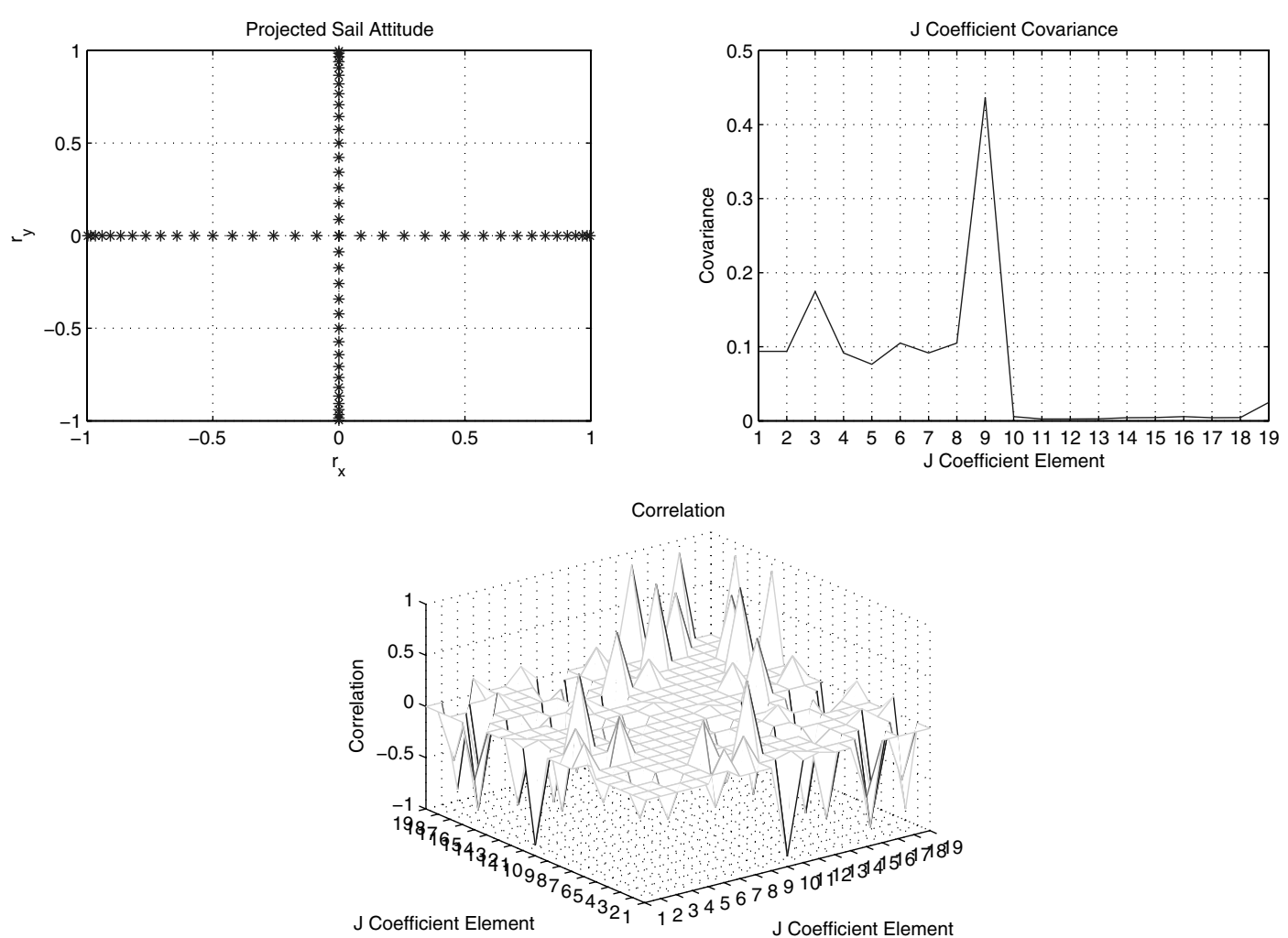

Fig. 2 Attitude, covariance, and correlation, case II.

measuring units). The projected sail attitude is a plot of the $y$ component versus the $x$ component of $\hat{\mathbf{r}}$ on the sail $x-y$ plane. The correlations of a given tensor coefficient with itself are suppressed from the plots because by definition they are unity. From these figures it can be seen that case III has the lowest covariance and correlation.
Figure 5 shows the force covariance for each of the four cases as obtained from Eq. (38). Cases I and II have similar force covariance along all axes. Cases I, II, and IV perform similarly along the $x$ axis. Along the $z$-axis case III performs better at high sun-sail angles $\alpha$.

All the cases have very similar results for the estimated force and comparable errors, showing that the estimation process converges

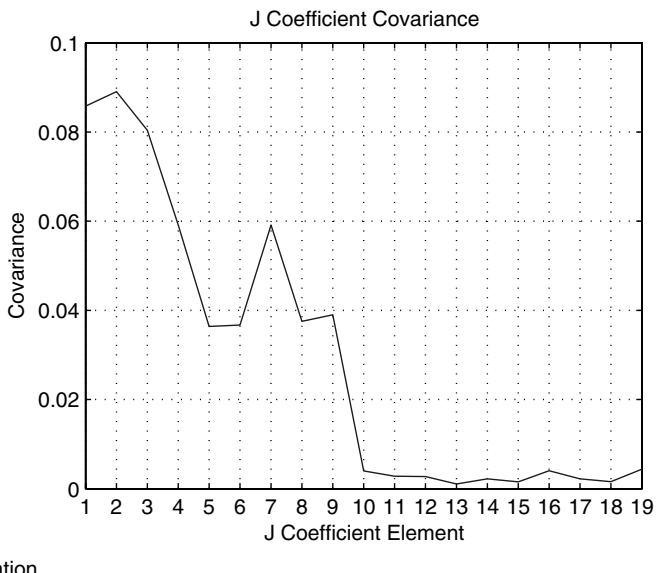

Correlation

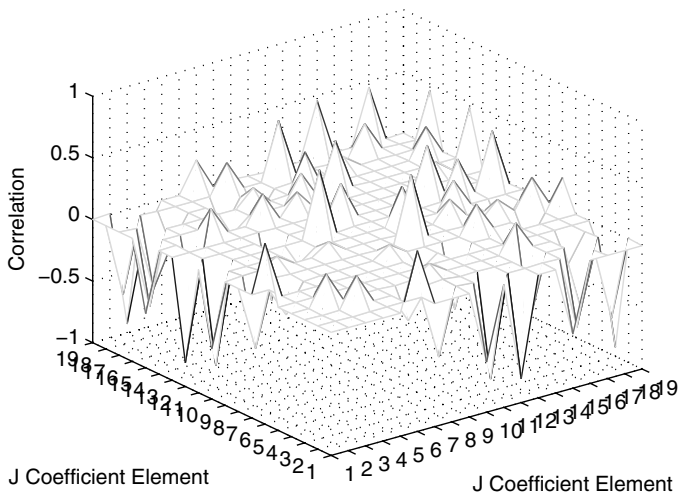

Fig. 3 Attitude, covariance, and correlation, case III. 

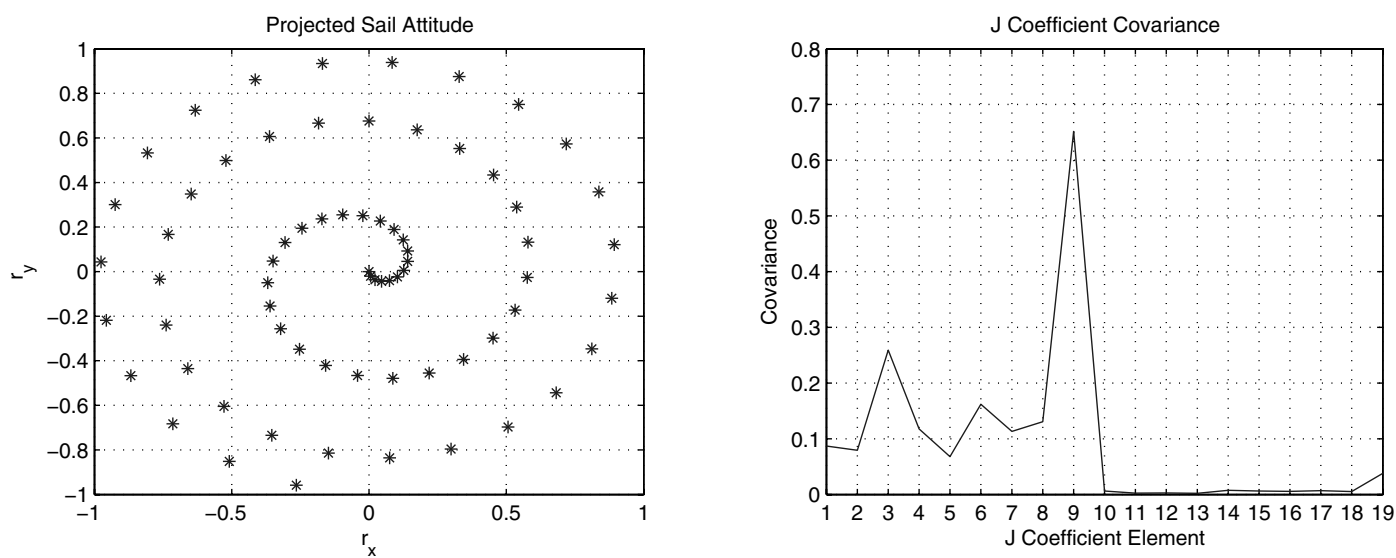

Correlation

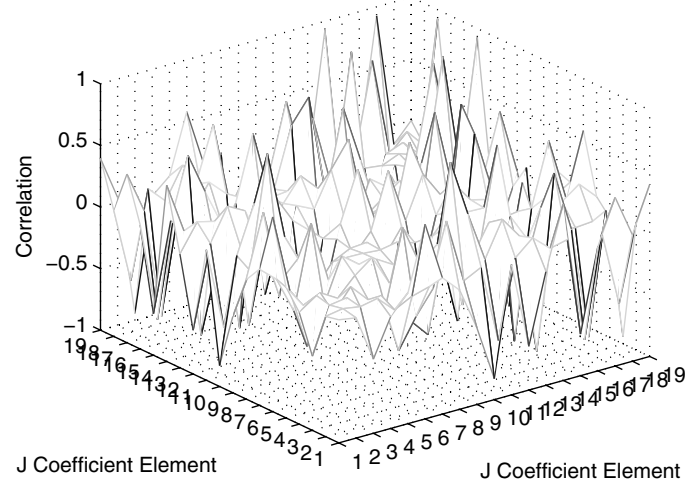

Fig. 4 Attitude, covariance, and correlation, case IV.

upon the correct values. Figure $\underline{6}$ shows representative results of the actual normalized force of the sinusoid sail and the estimated normalized force as well as their respective error.

\section{B. Moment Estimation}

The moment covariance and correlation were computed for measurements as in cases III and IV. Case I has measurements when $\hat{r}_{1}$ or $\hat{r}_{2}$ are zero and case II has measurements when $\hat{r}_{1}=\hat{r}_{2}$. Both situations make $\mathbf{A}_{M}$ rank deficient and $\Lambda_{M M}$ singular and were not used in the estimation.

The moment coefficients were estimated and the moment results for cases III and IV were compared with the actual moment generated by the sinusoid sail. The moment coefficients covariance and correlations for these cases are shown in Figs. 7-10. Case III has a
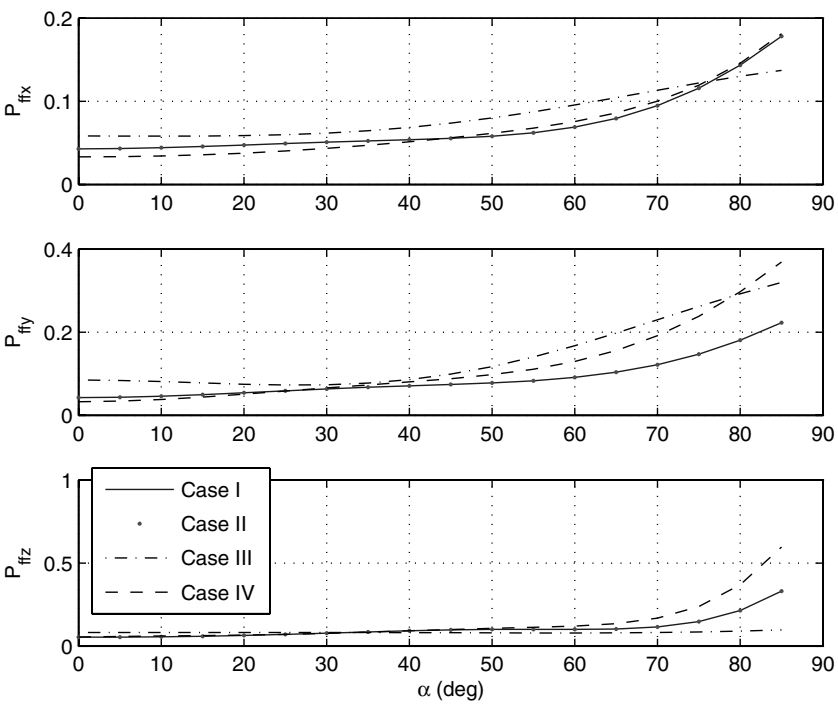

Fig. 5 Force covariance. better performance in the covariance and correlation of its estimated parameters. Both cases III and IV replicate the sail moment with comparable errors as shown in the representative in Fig. 11. This estimation was performed with 70 data measurements.

One last case was carried out by performing a uniform data sampling across all the sail possible attitudes. The sampling was done with 8100 data measurements. The moment covariance shrinks to zero. The estimation was successful in recovering the moment generated by the sail with small deviations from the actual values and slight improvements over the previous two cases.

\section{Conclusions}

In this paper a methodology for estimating the forces and moments acting on a sail of arbitrary shape is presented based on the
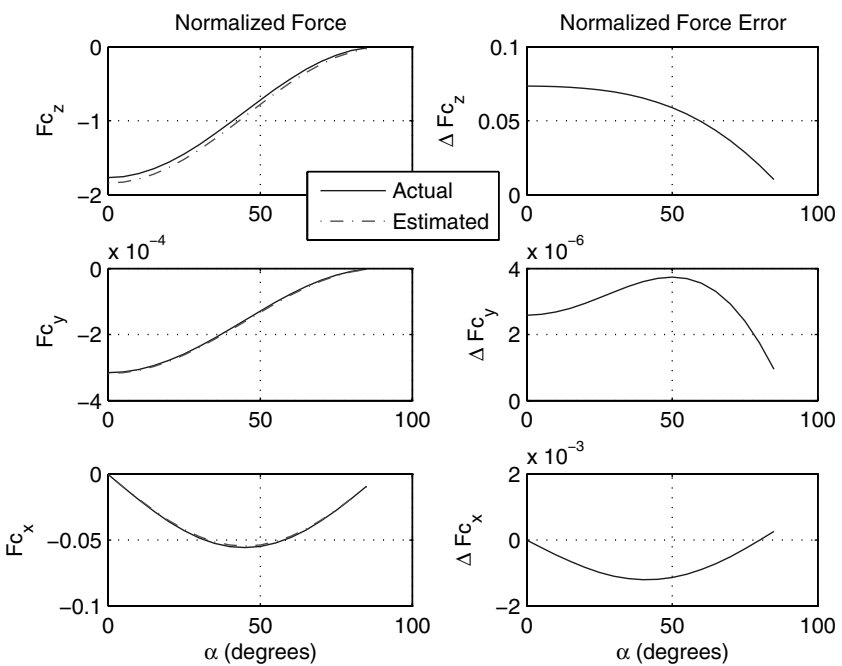

Fig. 6 Representative force estimation results. 


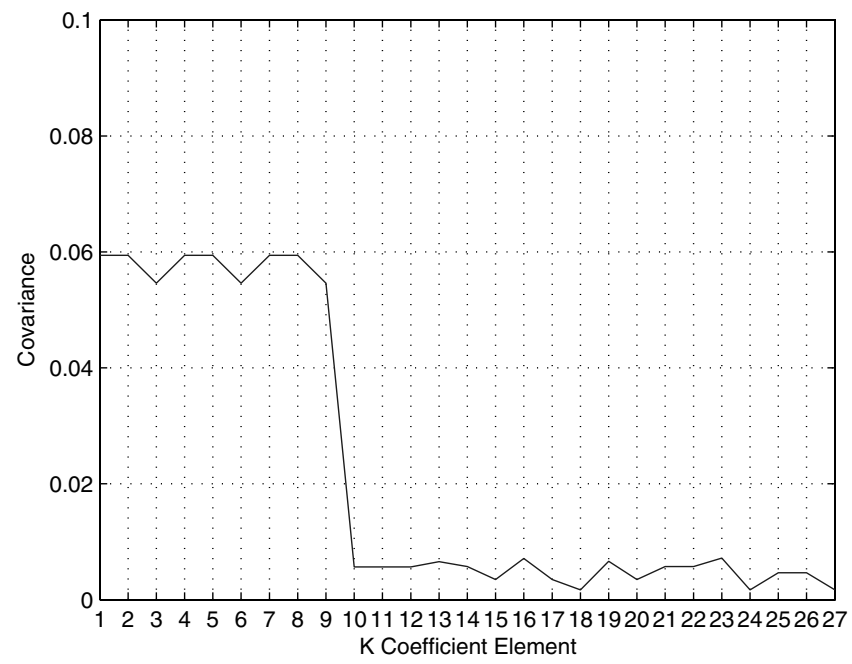

Fig. 7 Case III, moment coefficients covariance.

generalized sail model. The estimation is based on a least-squares algorithm. The accuracy of the estimation was dependent on the sail attitude relative to the sun when the measurement sampling was taken. The moment estimation is more sensitive to the data sampling and the attitudes to be avoided were discussed.

A nonsymmetric sinusoid sail shape was used to simulate force and moment data. Forces and moments were estimated based on these data. Four different cases of data samplings were used to estimate the forces. All four cases recovered the force from the sinusoid sail with small errors. The moment tensor coefficients were also estimated using a least-squares algorithm.

The estimation of both the force and moment included the optical parameters in the definition of the tensor coefficients, which made it possible to estimate their combination. Because the tensor coefficients and optical parameters appear as a product there is no need to estimate them separately.

\section{Appendix: General Sail Shape}

In this Appendix we define a general, square, nonsymmetric sail. This sail geometry is used to generate force and moment data needed for the estimation of the GSM coefficients. To generate the sail shape

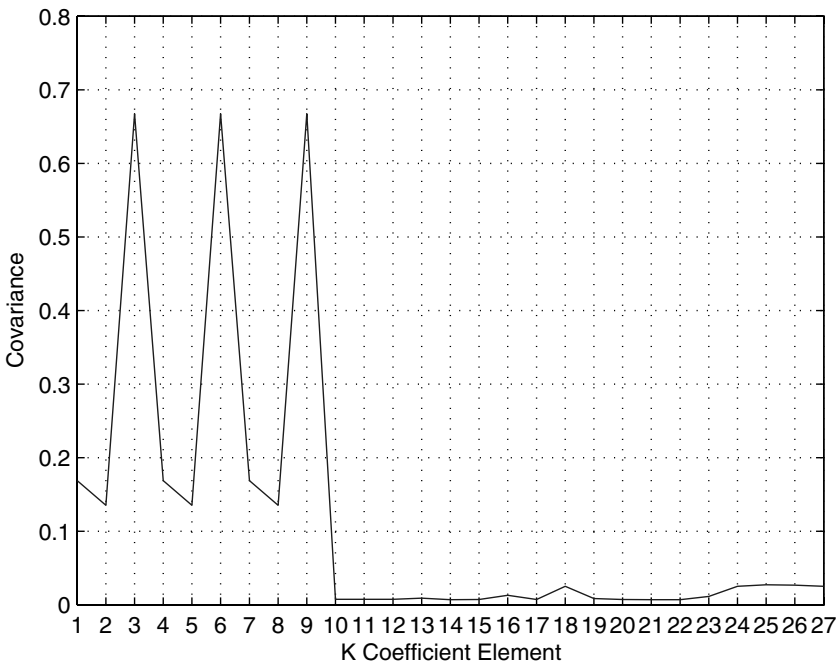

Fig. 9 Case IV, moment coefficients covariance.

a surface of the form is used:

$$
\begin{aligned}
z= & 1-\left[1+\cos \left(\frac{\pi x}{l}\right)+a_{x} \cos \left(\frac{f_{x} \pi x}{l}\right)+b_{x} \sin \left(\frac{2 \pi x}{l}\right)\right][1 \\
& \left.+\cos \left(\frac{\pi y}{l}\right)+a_{y} \cos \left(\frac{f_{y} \pi y}{l}\right)+b_{y} \sin \left(\frac{2 \pi y}{l}\right)\right]
\end{aligned}
$$

where $l$ is the length of one side of the sail, $a$ and $b$ are the amplitude of deformations along the $x$ and $y$ axes, and $f$ is the frequency. $a, b$, and $f$ allows one to change the sail shape easily, choosing values of $l=100 \mathrm{~m}, a_{x}=a_{y}=0.2, b_{x}=b_{y}=0$, and $f_{x}=f_{y}=5$. The sail shape for the values is shown in Fig. 12. Note that this is an arbitrary, but generally nonsymmetric shape, used for example purposes only.

This shape can be modified by assigning values to $b_{x}$ and $b_{y}$. The force and moment tensor coefficients were computed for this shape to be used in numerical experiments.

$$
\mathbf{J}^{1}=\left[\begin{array}{c}
0 \\
0 \\
1.0560 E-001
\end{array}\right]
$$

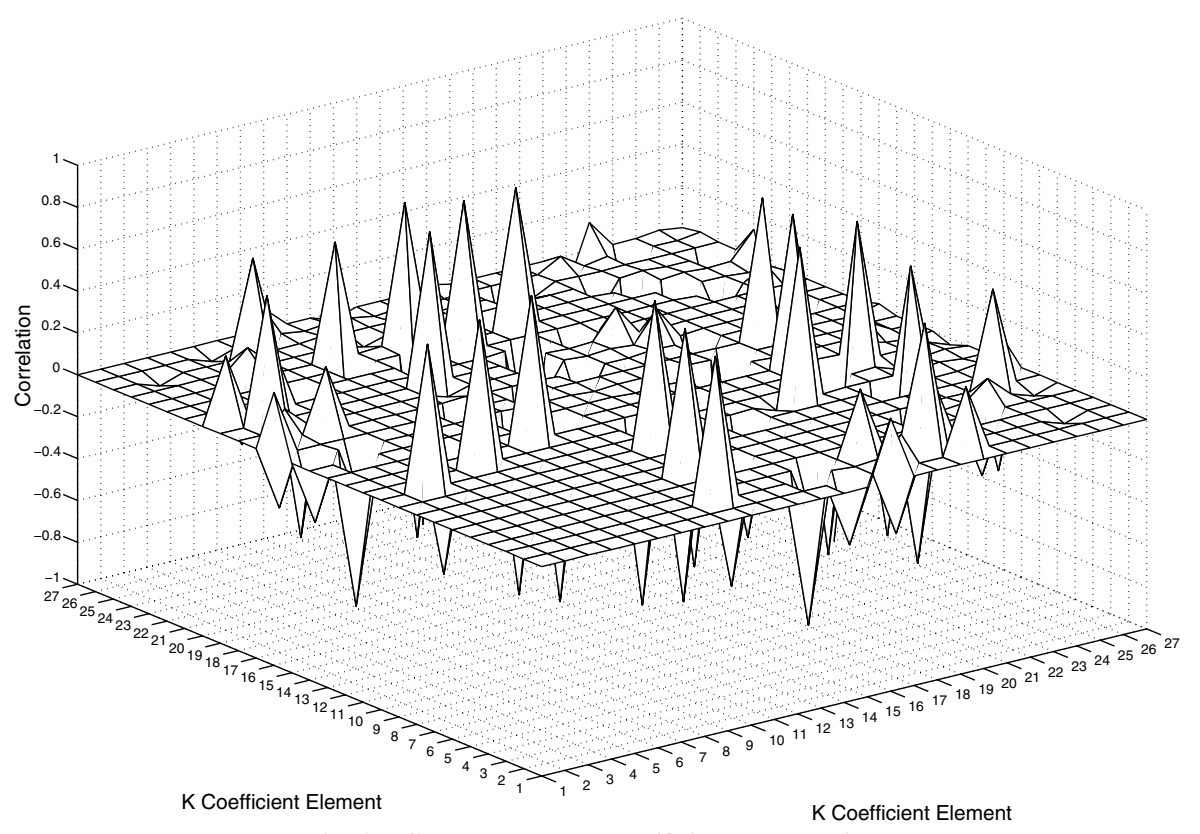

Fig. 8 Case III, moment coefficients correlation. 


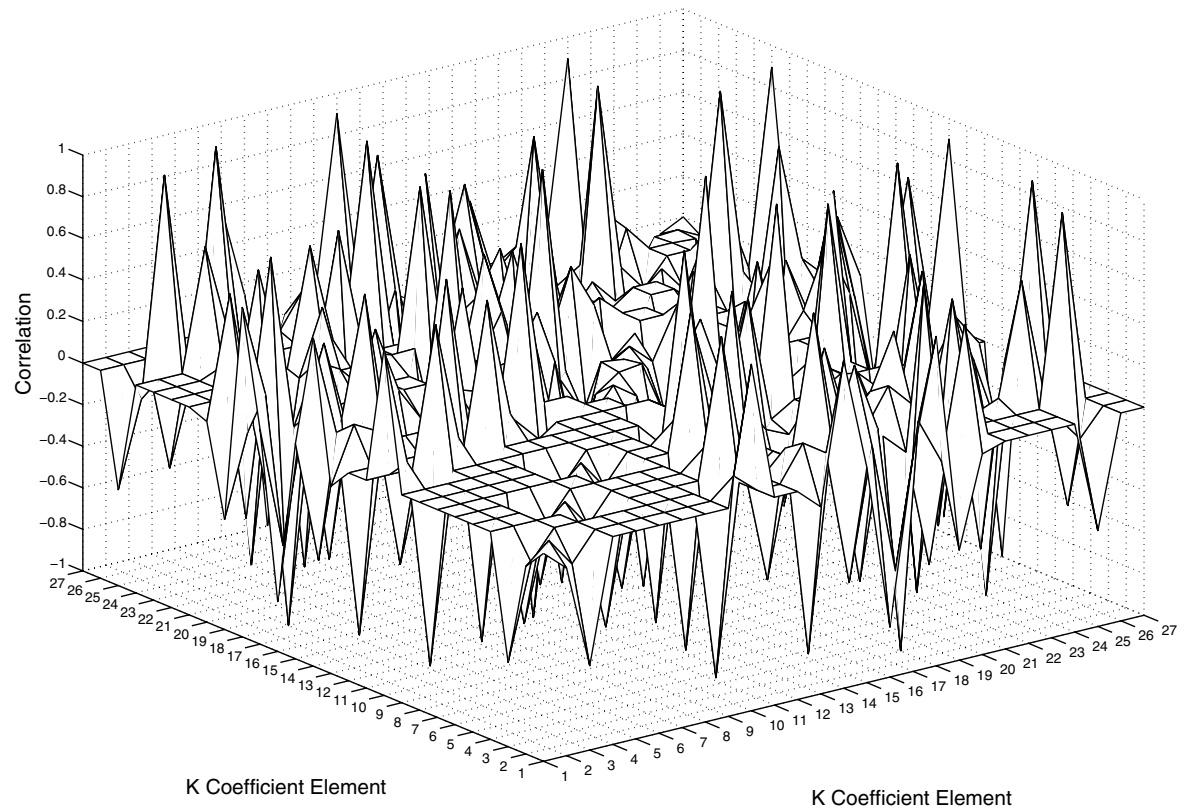

Fig. 10 Case IV, moment coefficients correlation.

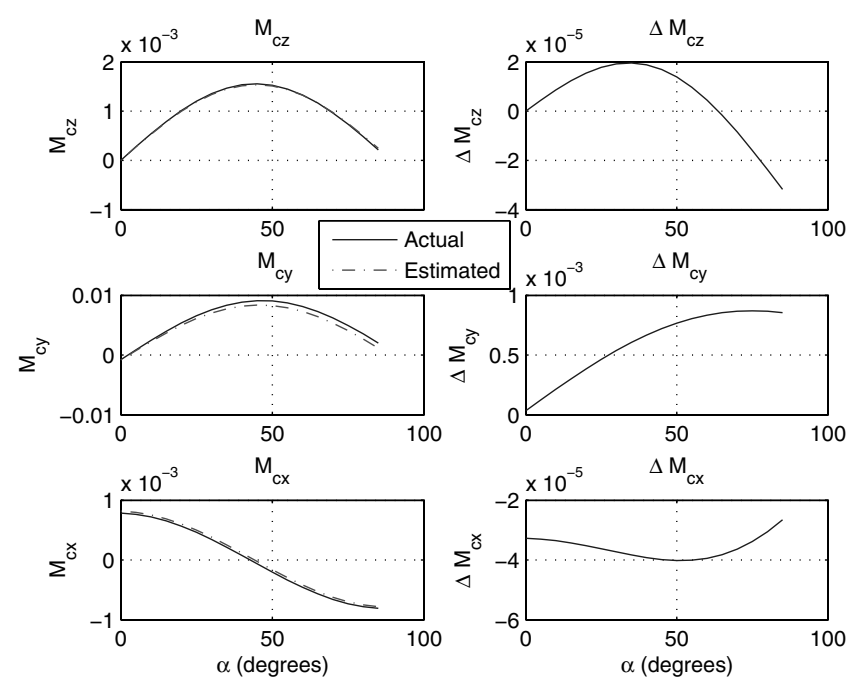

Fig. 11 Representative moment estimation results.

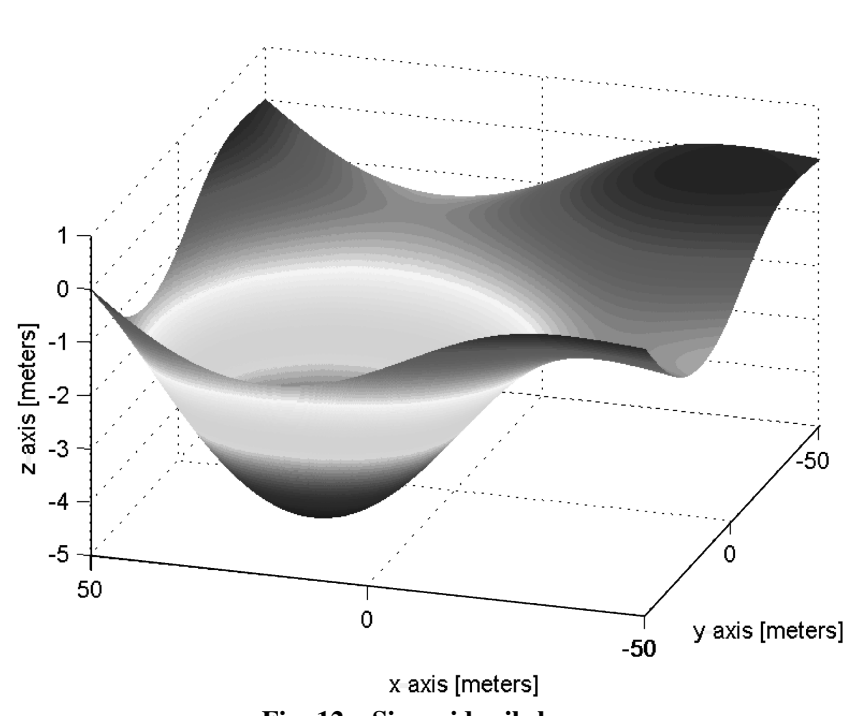

$\mathbf{J}^{2}$

$$
=\left[\begin{array}{ccc}
-1.7835 E-004 & 5.6948 E-010 & -3.9270 E-006 \\
5.6948 E-010 & -1.7835 E-004 & -3.9270 E-006 \\
-3.9270 E-006 & -3.9270 E-006 & -4.2180 E-002
\end{array}\right]
$$

$$
\begin{aligned}
& \mathbf{J}_{i j 1}^{3} \\
& \quad=\left[\begin{array}{ccc}
-3.2865 E-004 & -1.2754 E-007 & 7.4675 E-003 \\
-1.2754 E-007 & -1.2754 E-007 & -4.7843 E-008 \\
7.4675 E-003 & -4.7843 E-008 & 3.2878 E-004
\end{array}\right]
\end{aligned}
$$

$\mathbf{J}_{i j 2}^{3}$

$$
=\left[\begin{array}{ccc}
-1.2754 E-007 & -1.2754 E-007 & -4.7843 E-008 \\
-1.2754 E-007 & -3.2865 E-004 & 7.4675 E-003 \\
-4.7843 E-008 & 7.4675 E-003 & 3.2878 E-004
\end{array}\right]
$$

$$
\begin{aligned}
& \mathbf{J}_{i j 3}^{3} \\
& \quad=\left[\begin{array}{ccc}
7.4675 E-003 & -4.7843 E-008 & 3.2878 E-004 \\
-4.7843 E-008 & 7.4675 E-003 & 3.2878 E-004 \\
3.2878 E-004 & 3.2878 E-004 & 1.7739 E+000
\end{array}\right]
\end{aligned}
$$

$\mathbf{K}^{2}$

$$
=\left[\begin{array}{ccc}
-5.5317 E-007 & 4.3586 E-004 & 2.9039 E-005 \\
-4.3586 E-004 & 5.5317 E-007 & -2.9039 E-005 \\
2.0073 E-005 & -2.0073 E-005 & 1.2131 E-018
\end{array}\right]
$$

$$
\begin{aligned}
& \mathbf{K}_{i j 1}^{3} \\
& \quad=\left[\begin{array}{ccc}
-5.2438 E+000 & 1.0109 E-006 & -4.6226 E-005 \\
5.2447 E+000 & -1.0109 E-006 & -1.8246 E-002 \\
-4.6161 E-005 & -9.0270 E-019 & 8.3802 E-004
\end{array}\right]
\end{aligned}
$$




$$
\begin{aligned}
& \mathbf{K}_{i j 2}^{3} \\
& \quad=\left[\begin{array}{ccc}
1.0109 E-006 & -5.2446 E+000 & 1.8246 E-002 \\
-1.0109 E-006 & 5.2439 E+000 & 4.6226 E-005 \\
-9.0615 E-019 & 4.6161 E-005 & -8.3802 E-004
\end{array}\right]
\end{aligned}
$$

$$
\begin{aligned}
& \mathbf{K}_{i j 3}^{3} \\
& \quad=\left[\begin{array}{ccc}
-4.6226 E-005 & 1.8246 E-002 & -5.2405 E+000 \\
-1.8246 E-002 & 4.6226 E-005 & 5.2406 E+000 \\
8.3802 E-004 & -8.3802 E-004 & -1.8401 E-017
\end{array}\right]
\end{aligned}
$$

\section{Acknowledgement}

The authors would like to acknowledge the support for this research from the Jet Propulsion Laboratory, California Institute of Technology, which is under contract with the National Aeronautics and Space Administration.

\section{References}

[1] Lura, F., and Hagelschuer, D., "Experiments in the Test Facility Kobe for the Investigation of Degradation Effects of Thin Foil Samples for a Solar Sail Mission Concerning the Simultaneous Influence of Space Environment Properties," 22nd Space Simulation Conference, 2124 Oct. 2002.

[2] Rios-Reyes, L., and Scheeres, D. J., "Generalized Model for Solar Sails," Journal of Spacecraft and Rockets, Vol. 42, No. 1, 2005, pp. 182-185.

[3] Banik, J. A., Lively, P. S., Taleghani, B. K., and Jenkins, C. H., "Solar Sail Variations due to On-Orbit Thermal Effects," Paper 2006-1708, May 2006.

[4] Black. J. T., and Papa, R. S., "Photogrammetry and Videogrammetry Methods for Solar Sails and Other Gossamer Structures," Paper 20041662, April 2004.

[5] McInnes, C. R., Solar Sailing: Technology, Dynamics and Mission Applications, 1st ed., Springer-Praxis, Chichester, U.K., 1999, pp. $43-$ 49.

[6] Derbes, B., and Lichodziejewski, D., "Sail Coordinate Systems and Format for Reporting Propulsive Perfomance," AAS Paper AAS 04100, Feb. 2004.

[7] Koch, K. R., Parameter Estimation and Hypothesis Testing in Linear Models, Springer, New York, 1999, pp. 152-163. 